\title{
Disección endoscópica submucosa de tumor neuroendocrino primario de duodeno. Reporte de caso y revisión de la literatura
}

\author{
Martín Edgardo Rojano Rodríguez, ${ }^{1}$ Elisafat Arce Liévano, ${ }^{1}$ Orlando Bada Yllán, ${ }^{1}$ \\ Carlos Valenzuela Salazar, ${ }^{1}$ Roberto Délano Alonso, ${ }^{1}$ Elio Germán Recinos Carrera, ${ }^{2}$ \\ José de Jesús Herrera Esquivel ${ }^{1}$
}

${ }^{1}$ Departamento de Endoscopía Gastrointestinal, Hospital General "Dr. Manuel Gea González", SSA, Ciudad de México, México.

${ }^{2}$ Departamento de Anatomía Patológica, Hospital General "Dr. Manuel Gea González", SSA, Ciudad de México, México.

Acta Gastroenterol Latinoam 2020;50(2):177-182

Recibido: 11/01/2019 / Aceptado: 27/01/2020 / Publicado online: 29/06/2020

\section{Resumen}

Introducción. Los tumores neuroendocrinos primarios de duodeno son neoplasias muy infrecuentes; cuando están limitados a la mucosa y no tienen metástasis ganglionares pueden ser resecados de manera endoscópica con intención curativa. Caso clínico. Presentamos el caso de una paciente mexicana de 52 años con tumor neuroendocrino primario de duodeno, que fue sometida a disección endoscópica de la submucosa obteniéndose márgenes libres de tumor (RO) y que evolucionó satisfactoriamente, siendo egresada a su domicilio a las 24 horas posprocedimiento. Las biopsias tomadas durante el seguimiento endoscópico a las cuatro semanas reportaron tejido de granulación con estroma de tejido fibroso en lámina propia, sin evidencia de neoplasia maligna. Conclusiones.

Correspondencia: Elisafat Arce Liévano

Emiliano Zapata 62, Interior GHO2 (CP 03300). Colonia Portales Sur, Delegación Benito Juárez, Ciudad de México, México Tel.: 015540003000 (Ext. 3329) / Cel.: 0445537176302

Correo electrónico:dr.elisafat.arce@gmail.com
Debido a su baja frecuencia, no existen guias de manejo universalmente aceptadas para los tumores neuroendocrinos primarios de duodeno, y su tratamiento es controversial. En general, para tumores menores de $1 \mathrm{~cm}$ se recomienda la resección endoscópica y para aquellos mayores de $2 \mathrm{~cm}$ se recomienda resección quirúrgica. El manejo de tumores entre 1 y $2 \mathrm{~cm}$ es el más controversial y se recomienda individualizarlo en cada caso.

Palabras claves. Tumor neuroendocrino duodenal, resección endoscópica de la mucosa, disección endoscópica de la submucosa.

\section{Endoscopic submucosal dissection of duodenal neuroendocrine tumour. Case report}

\section{Summary}

Background. Duodenal neuroendocrine tumours are very rare, if they are limited to the mucosal layer and have no nodal involvement, endoscopic resection may be curative. Case report. We present the case of a Mexican 52 years old female with a duodenal neuroendocrine tumour who underwent successful endoscopic submucosal dissection with no complications. Pathology of the specimen revealed a complete $R 0$ resection with negative resection margins. Endoscopic biopsies 4 weeks later showed no malignant cells. Conclusions. Because of their low frequency the management of primary duodenal carcinoid tumours is controversial; for tumours less 
than $1 \mathrm{~cm}$ endoscopic resection is recommended, for those bigger than $2 \mathrm{~cm}$ surgical resection is advised. Management of tumours between 1 and $2 \mathrm{~cm}$ is controversial and should be individualized.

Key words. Duodenal neuroendocrine tumour, endoscopic mucosal resection, endoscopic submucosal dissection.

\section{Abreviaturas}

TNE: Tumores neuroendocrinos.

DTNE: Tumores neuroendocrinos primarios de duodeno.

USE: Ultrasonido endoscópico.

NBI: Narrow band imaging.

DES: Disección endoscópica de la submucosa.

NEM-1: Neoplasia endócrina múltiple tipo 1.

ENETS: Sociedad Europea de Tumores Neuroendocrinos.

NCCN: National Comprehensive Cancer Network.

REM: Resección endoscópica de la mucosa.

\section{Introducción}

Los tumores carcinoides forman parte de la familia de tumores neuroendócrinos (TNE) que se originan de las células enterocromafines. ${ }^{1}$ Las localizaciones más comunes son el recto $(17,7 \%)$, el intestino delgado $(17,1 \%$, la mayoría en el íleon) y el colon $(10 \%)$. $^{2}$ Los tumores neuroendocrinos primarios de duodeno (DTNE) son infrecuentes, representan el 2,9\% del total de TNE del tracto gastrointestinal; suelen ser asintomáticos y se descubren de manera incidental en una endoscopíaa realizada por otras indicaciones. ${ }^{1,3}$ Los DTNE son más comunes en hombres; la mayoría son únicos y el 75\% son menores de $2 \mathrm{~cm} .{ }^{4,5}$ El 90\% de los DTNE son no funcionantes. ${ }^{6}$

Los carcinomas neuroendocrinos primarios de duodeno son neoplasias malignas que presentan metástasis ganglionares en etapas tempranas. La incidencia descrita de invasión ganglionar de los carcinoides neuroendocrinos de duodeno menores a $1 \mathrm{~cm}$ de longitud varía entre 4,5\% y $13,2 \% .{ }^{1,7}$ Es precisamente por este curso clínico agresivo por lo que actualmente está indicada su resección, independientemente del tamaño. ${ }^{1,2}$

El diagnóstico de DTNE se realiza histológicamente mediante inmunohistoquímica positiva para Cromogranina A, sinaptofisina, enolasa, CD-56 y Ki-67.4 El ultrasonido endoscópico (USE) es una herramienta muy útil para determinar el tamaño, el grado de invasión del tumor y la presencia o ausencia de metástasis ganglionares para poder planear el abordaje ideal para su resección (resección quirúrgica vs. resección endoscópica). ${ }^{14}$

\section{Caso clínico}

Se presenta el caso de una paciente femenina de 52 años sin antecedentes de importancia, a la que se le realizó una endoscopía superior por presentar síntomas de dispepsia refractarios a tratamiento médico. Durante la revisión endoscópica, a nivel de la primera porción de duodeno se identificó una lesión subepitelial de aproximadamente 10-12 mm sin presencia de erosión central; a la aplicación de cromoendoscopía digital (NBI Olympus, Tokyo, Japón) y Near Focus presentó mucosa con criptas de características gástricas (Figura 1).

Se tomaron biopsias con reporte histopatológico de neoplasia neuroendocrina maligna bien diferenciada grado 1 (Ki-67 del 2\%, CD56 +++/+++, Cromogranina A $+++/+++$, sinaptofisina $+++/+++$ ) (Figura 2$)$. Se comple-

Figura 1. A) Visión endoscópica con luz blanca de tumor carcinoide en bulbo duodenal, B) visión endoscópica con cromoendoscopia digital (Narrow Band Imaging; NBI) de tumor carcinoide en bulbo duodenal.

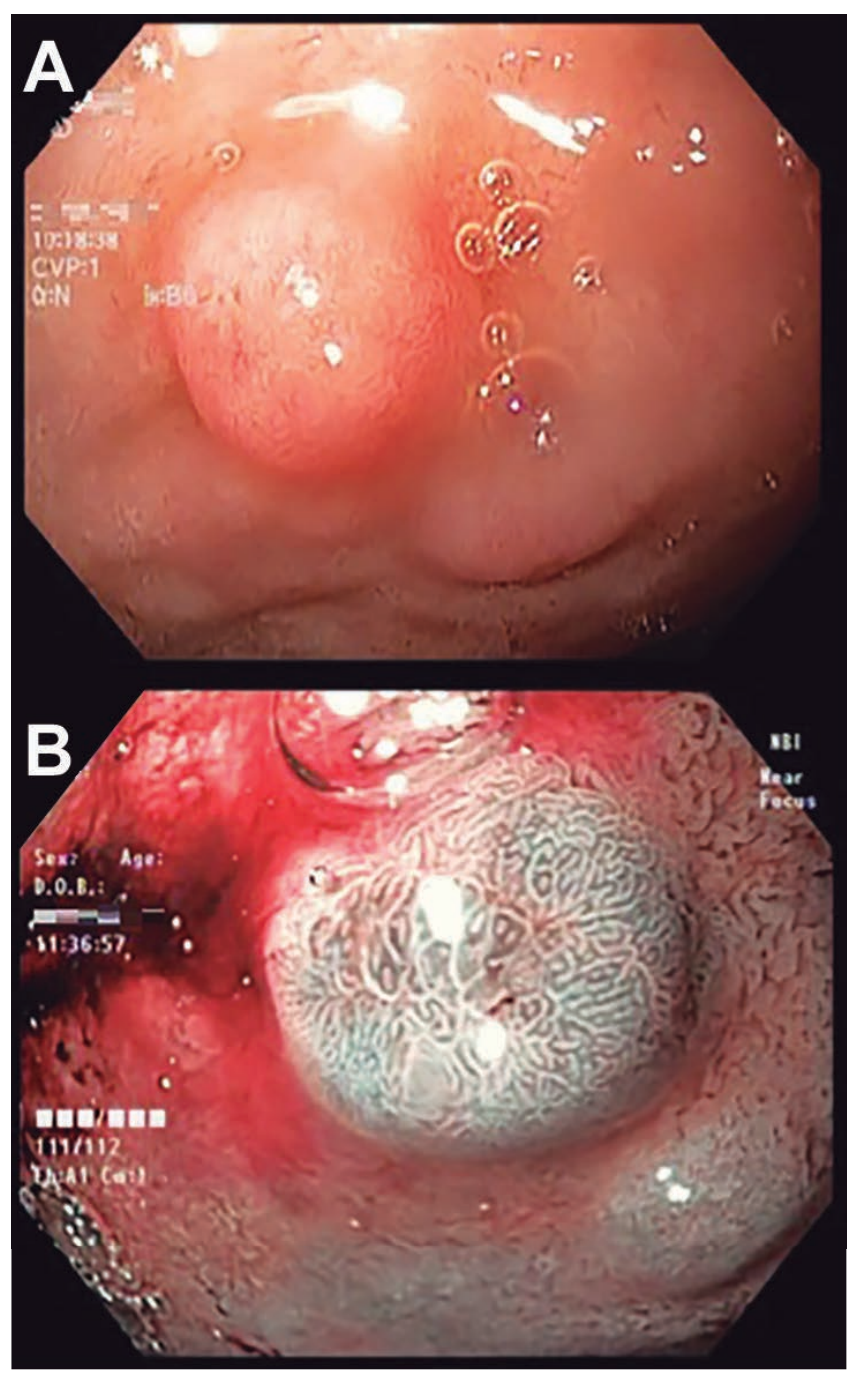


Figura 2. A) Fotomicrografía con Hematoxilina y Eosina a $4 X$ que muestra nidos sólidos de neoplasia neuroendocrina maligna (flecha), B) inmunohistoquimica para CD56 positivo a 40X, C) inmunohistoquimica para Cromogranina positivo a $40 X$, D) inmunohistoquimica para sinaptofisina positivo a $40 X$.

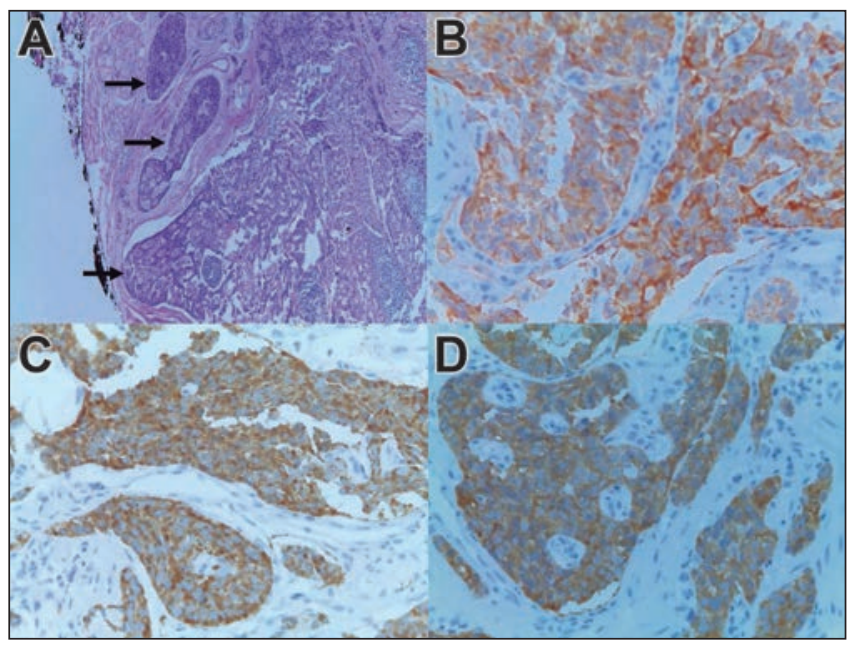

mentó abordaje con la realización de USE, el cual reportó tumor de bordes regulares de $10 \mathrm{~mm}$ de diámetro mayor dependiente de la submucosa del duodeno que respeta la capa muscular propia, sin presencia de invasión linfática (Figura 3), por lo que se le realizó disección endoscópica submucosa (DES) de la lesión mediante la siguiente técnica:

Con paciente en sala de quirófano y en decúbito late-

Figura 3. Ultrasonido Endoscópico que demuestra imagen hipoecogénica de $10 \mathrm{~mm}$ de diámetro mayor.

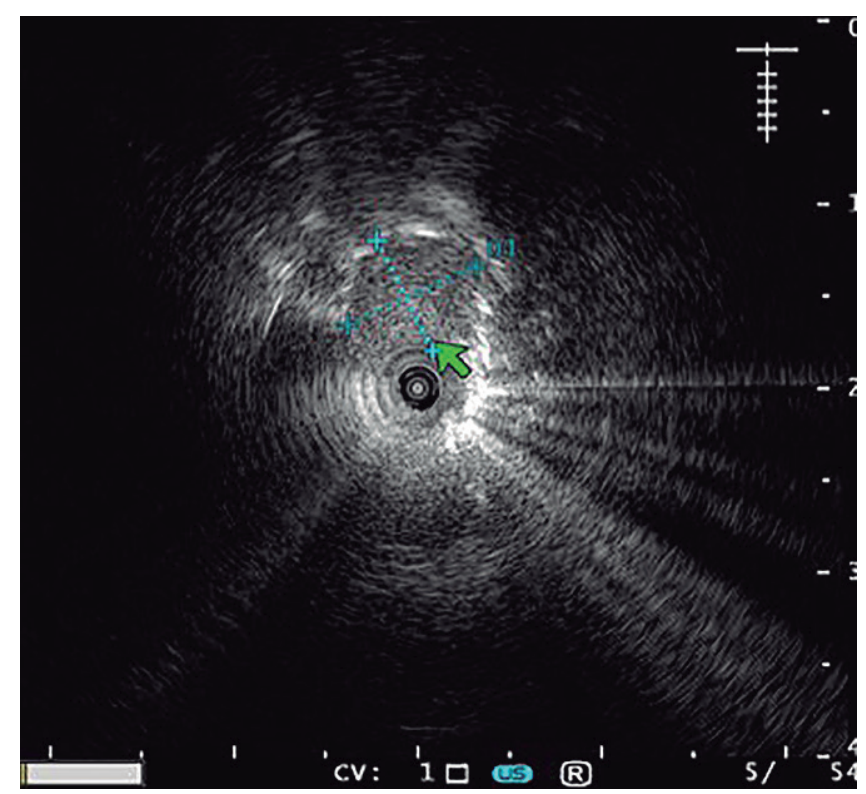

ral izquierdo, previa administración de cefalosporina de segunda generación 30 minutos previos al procedimiento y bajo anestesia general e intubación endotraqueal a cargo del servicio de anestesiología, se introdujo videoendoscopio GIF-Q180 (Olympus, Tokyo, Japón) hasta bulbo duodenal. Posteriormente se utilizó una navaja con punta aislada "IT-knife" (Olympus, Tokyo, Japón) y una unidad electroquirúrgica ERBE VIO 300D (ERBE Elektromedizin, Turbingen, Alemania) programada con parámetros de $100 \mathrm{~W}$, efecto 5 y coagulación suave para realizar un marcaje circunferencial en la mucosa sana 3 $\mathrm{mm}$ por fuera de la lesión a resecar. Una vez marcados los límites, se realizó inyección submucosa de la lesión con aguja calibre 25 Gauge iniciando por el borde proximal con $2 \mathrm{ml}$ de ácido hialurónico y $1 \mathrm{ml}$ de índigo carmín al $0,25 \%(0,5 \mathrm{ml}$ en $500 \mathrm{ml})$, observándose una adecuada elevación de la lesión, a excepción del sitio donde se tomó la biopsia para realizar el diagnóstico de DTNE. Posteriormente se realizó una incisión transversal con una aguja de corte "needle-knife" (Olympus, Tokyo, Japón) y se introdujo el "IT-knife" para ampliarla; se colocó un capuchón en la punta distal del endoscopio, el cual se utilizó para presentar el tejido submucoso y realizar tracción de la pieza para mantener el plano de disección. Para la disección del lecho de la lesión se utilizó una combinación de navaja de gancho "Hook knife", navaja de punta aislada "IT-knife" y navaja de triángulo "triangle tip knife" (Olympus, Tokyo, Japón) con parámetros de la unidad electroquirúrgica ajustados a modo coagulación forzada y $40 \mathrm{~W}$; durante este paso los vasos submucosos que se iban exponiendo fueron coagulados utilizando una sonda bipolar "BiCOAG bipolar probe" y una pinza hemostática "Coagrasper" (Olympus, Tokyo, Japón) para prevenir un posible sangrado asociado a la DES y mantener el campo limpio. Finalmente, se colocaron clips hemostáticos "Resolution $^{\text {TM" }}$ (Boston Scientific, EE. UU.) en el sitio de disección para afrontar la mucosa (Figura 4); se extrajo la pieza con ayuda de una canastilla, se fijó y orientó para ser enviada a patología. La paciente fue extubada sin complicaciones e ingresada a piso para vigilar su evolución. Se inició dieta a las seis horas posteriores al evento endoscópico y fue egresada a su domicilio asintomática y sin complicaciones a las 24 horas. El reporte de patología fue de resección completa ("R0") de neoplasia neuroendocrina maligna bien diferenciada grado 1 (Ki-67 del 2\%) con bordes laterales y lecho libres de tumor (Figura 5). Como parte del seguimiento se realizó endoscopía superior con toma de biopsias del sitio de resección a las cuatro semanas; las biopsiasreportaron tejido de granulación con estroma de tejido fibroso en lámina propia, sin evidencia de neoplasia maligna. 
Figura 4. Disección endoscópica submucosa de tumor carcinoide duodenal. A) Marcaje de los límites de la lesión a resecar, B) inyección de la submucosa con ácido hialurónico, $\boldsymbol{C} \boldsymbol{y}$ D) disección de la capa submucosa con IT-Knife y capuchón, E) lecho quirúrgico, $\boldsymbol{F}$ ) colocación de clips hemostáticos en el sitio de disección.

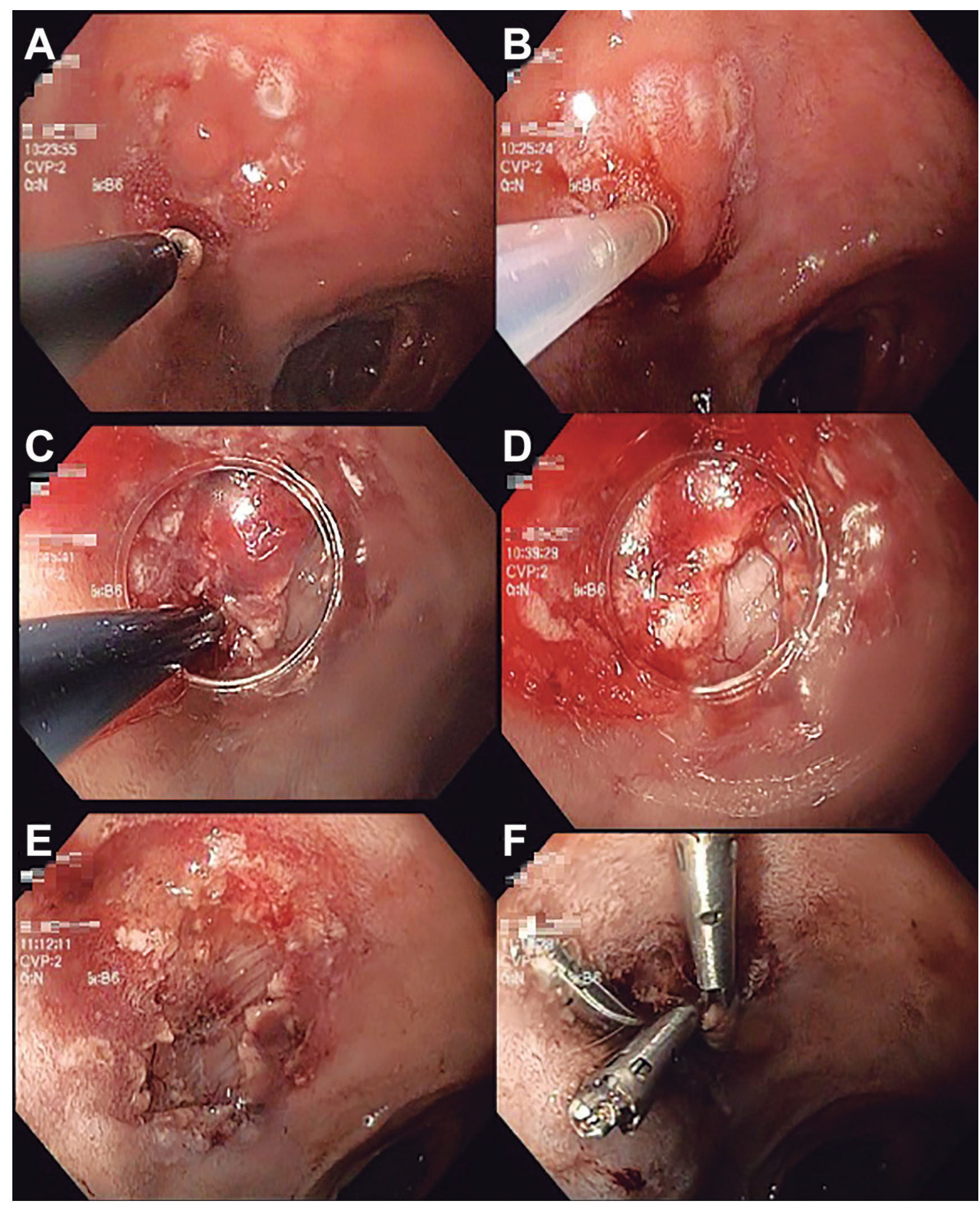


Figura 5. UFotomicrografía con Hematoxilina y Eosina a 40X que muestra células neoplásicas (flechas) y glándulas de Brunner sin infiltración (asteriscos).

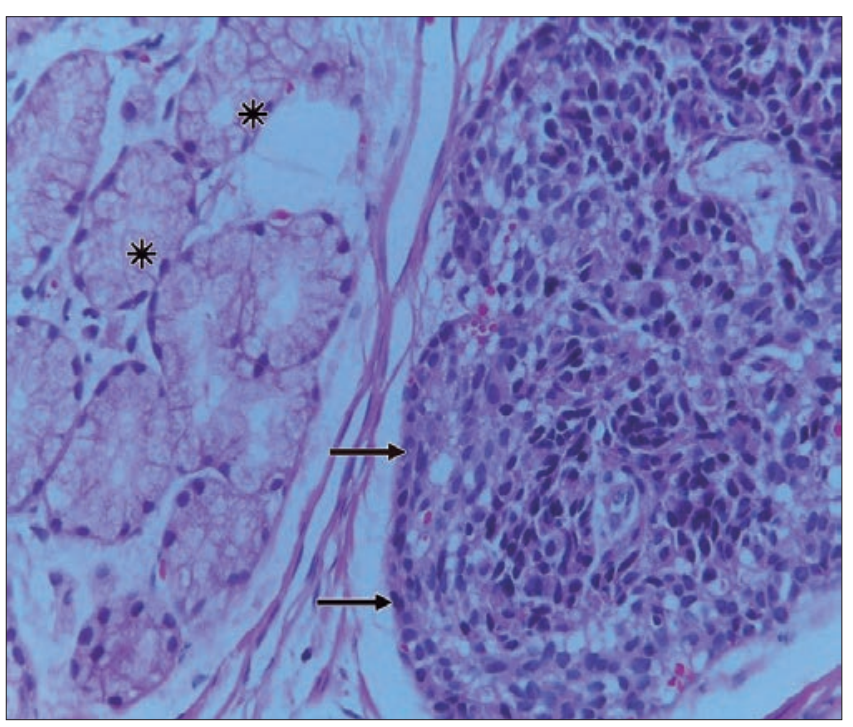

\section{Discusión}

A diferencia de los TNE en otras localizaciones del tracto gastrointestinal (estómago, apéndice, recto, etc.) el tratamiento de los DTNE es controversial y no existen guías de manejo universalmente aceptadas. ${ }^{1}$

Las guías de la Sociedad Europea de Tumores Neuroendocrinos (ENETS, por sus siglas en inglés) recomiendan que los DTNE menores a $1 \mathrm{~cm}$, bien diferenciados, sin presencia de invasión linfática o a distancia, en localizaciones no ampulares, sean tratados mediante resección endoscópica. ${ }^{8}$ Para el manejo de los DTNE mayores a $2 \mathrm{~cm}$ se recomienda la resección quirúrgica. El manejo para los DTNE de entre 1 y $2 \mathrm{~cm}$ es el más controversial y se recomienda individualizar cada caso. 8

En los DNTE de entre 1 y $2 \mathrm{~cm}$ debe determinarse mediante ultrasonido endoscópico si existe compromiso ganglionar, ya que si este es positivo la resección completa (tumor primario y ganglios) únicamente puede lograrse mediante intervención quirúrgica con linfadenectomía. En el caso presentado en este artículo se determinó la ausencia de metástasis ganglionares, por lo que fue posible brindar un tratamiento endoscópico.

Existen diversas técnicas para lograr la resección en bloque de los DTNE que son candidatos a tratamiento endoscópico; la resección endoscópica de la mucosa (REM) es una técnica relativamente simple, eficaz y segura para la resección de tumores menores de $1 \mathrm{~cm}$; $\sin$ embargo para tumores de mayor diámetro la disección endoscópica de la submucosa (DES) ofrece mayor posibilidad de lograr una resección en bloque., 6

Las dos principales complicaciones de la DES son la hemorragia y la perforación. La primera se presenta en un 13 a 38\% de los pacientes y puede ser inmediata (primeras 24 horas) o tardía (posterior a 24 horas); suele ser autolimitada pero en ocasiones requiere soporte transfusional y tratamiento endoscópico urgente. La tasa de perforación descrita para DES es de 5\%, aunque en series occidentales donde el desarrollo de la técnica cuenta con menos experiencia se puede elevar hasta un $20 \%$; en caso de que la perforación no pueda ser reparada mediante aplicación endoscópica de clips metálicos o mecanismos de sutura, el procedimiento debe suspenderse y realizarse una reparación quirúrgica de la perforación. ${ }^{5,9}$ La realización del procedimiento por un endoscopista experimentado en la técnica, la inyección submucosa de un adecuado volumen que permita elevar la lesión y el uso de índigo carmín se han propuesto como medidas estándar para disminuir la frecuencia de perforaciones. ${ }^{9}$

En cuanto al caso específico que se presenta en este artículo, se optó por realizar DES ya que si bien el tumor tenía un tamaño limítrofe para su resección mediante REM, al momento de la inyección de la submucosa no se logró una adecuada elevación de la mucosa del borde distal (sitio donde se tomó la biopsia), por lo que obtener una resección completa "R0" hubiera sido menos probable mediante esta técnica. Si bien la DES conlleva un mayor riesgo de hemorragia y perforación, es importante mencionar que el procedimiento se realizó en un ambiente controlado (quirófano y con intubación endotraqueal) $y$ en una paciente con bajo riesgo quirúrgico (joven y sin comorbilidades). Además, dentro del equipo de trabajo contamos con la colaboración de cirujanos de mínima invasión, quienes estuvieron presentes durante el procedimiento para que en caso de que se presentara alguna complicación (sangrado o perforación) no susceptible de manejo endoscópico se resolviera mediante cirugía laparoscópica en el mismo evento anestésico. De tal forma que en este caso en particular los autores consideramos que el realizar una DES tenía mayor beneficio (resección $\mathrm{RO}$; curativa) que riesgo.

\section{Conclusión}

Como mencionamos anteriormente, la baja frecuencia de tumores neuroendocrinos primarios de duodeno ocasiona que la experiencia en el manejo de estos sea escasa si se compara con los TNE de otras localizaciones en el tracto gastrointestinal. Es por ello que las guías de manejo actuales tienen ciertas limitantes y son debatibles.

El manejo de la lesión que presentamos en este caso 
resulta particularmente controversial debido al diámetro limítrofe de esta. La resolución dada por los autores mediante una disección endoscópica de la mucosa resultó segura y efectiva en el seguimiento a cuatro semanas, por lo que consideramos que la DES realizada por un endoscopista experto y en casos seleccionados debe considerarse dentro de las opciones terapéuticas en pacientes con DTNE de entre 1 y $2 \mathrm{~cm}$.

Conflicto de intereses. Los autores declaran no tener ningún conflicto de intereses.

Sostén Financiero. La presente investigación no ha recibido ayudas especificas provenientes de agencias del sector público, comercial o entidades, y se realizó sin ánimo de lucro.

\section{Referencias}

1. Dogeas E, Cameron J, Wolfgang C, Hirose K, Hruban R, Makary M, Pawlik T, Choti M. Duodenal and Ampullary Carcinoid Tumors: Size Predicts Necessity for Lymphadenectomy. J Gastrointest Surg 2017; 25: 1262-1269.

2. Harnden I, Walker R, Balmadrid B, Jiang X, Obando J, Jowell P, Burbridge R. Endoscopic Mucosal Resection of Duodenal Carcinoid Tumors: A single Tertiary Care Center Experience. Gastroenterol Hepatol Open Access 2015; 3: 1-5.
3. Harada H, Suehiro S, Shimizu T, Katsuyama Y, Hayasaka K, Ito $H$. Ligation-assisted endoscopic submucosal resection with circumferential mucosal incision for duodenal carcinoid tumor. World J Gastroenterol 2015; 21: 10041-10044.

4. Mairena-Valle M. Carcinoide duodenal: reporte de caso. Endoscopía 2016; 28: 181-183.

5. Sato Y, Hashimoto S, Mizuno K, Takeuchi M, Terai S. Management of gastric and duodenal neuroendocrine tumors. World J Gastroenterol 2016; 22: 6817- 6828.

6. Randle R, Ahmed S, Newman N, Clark C. Clinical outcomes for neuroendocrine tumors of the duodenuma and ampulla of Vater: a population-based study. J Gastrointest Surg 2014; 18 : 354-362.

7. Soga J. Endocrinocarcinomas (carcinoids and their variants) of the duodenum. An evaluation of 927 cases. J Exp Clin Cancer Res 2003; 22: 349-363.

8. Delle F, Kwekkeboom D, Van E, Rindi G, Kos-Kudla B, Knigge U, Sasano H, Tomassetti P, Salazar R, Ruszniewski P. ENETS Consensus Guidelines for the management of patientis with gastroduodenal neoplasms. Neuroendocinology 2012; 95: 74-87.

9. Pérez D. Disección submucosa endoscópica ¿Solo para endoscopistas expertos?. Gastroenterol Hepatol 2012; 35 (5): 344-367. 